\title{
Treatment in a specialised out-patient mood disorder clinic $v$. standard out-patient treatment in the early course of bipolar disorder: randomised clinical trial ${ }^{\dagger}$
}

Lars Vedel Kessing, Hanne Vibe Hansen, Anne Hvenegaard, Ellen Margrethe Christensen, Henrik Dam, Christian Gluud, Jørn Wetterslev and The Early Intervention Affective Disorders (EIA) Trial Group

\section{Background}

Little is known about whether treatment in a specialised out-patient mood disorder clinic improves long-term prognosis for patients discharged from initial psychiatric hospital admissions for bipolar disorder
Aims
To assess the effect of treatment in a specialised out-patient mood disorder clinic $v$. standard decentralised psychiatric treatment among patients discharged from one of their first three psychiatric hospital admissions for bipolar disorder.

\section{Method}
Patients discharged from their first, second or third hospital admission with a single manic episode or bipolar disorder were randomised to treatment in a specialised out-patient mood disorder clinic or standard care (ClinicalTrials.gov: NCT00253071). The primary outcome measure was readmission to hospital, which was obtained from the Danish Psychiatric Central Register.

\section{Results}

A total of 158 patients with mania/bipolar disorder were included. The rate of readmission to hospital was significantly decreased for patients treated in the mood disorder clinic compared with standard treatment (unadjusted hazard ratio $0.60,95 \% \mathrm{Cl} 0.37-0.97, P=0.034$ ). Patients treated in the mood disorder clinic more often used a mood stabiliser or an antipsychotic and satisfaction with treatment was more prevalent than among patients who received standard care.

\section{Conclusions}

Treatment in a specialised mood disorder clinic early in the course of bipolar disorder substantially reduces readmission to a psychiatric hospital and increases satisfaction with care.

\section{Declaration of interest}

L.V.K. has been a consultant for Bristol-Myers Squibb, Eli Lilly, Lundbeck, Astrazeneca, Pfizer, Wyeth, Servier, and Janssen-Cilag.
Bipolar disorder is associated with a high risk of relapse and the risk of relapse increases as the number of previous episodes increases. ${ }^{1,2}$ Many patients do not recover to previous psychosocial function; ${ }^{3}$ some patients also present with cognitive impairment during the remitted phase ${ }^{4}$ and the risk of developing dementia may be increased in the long run. ${ }^{5}$ The tendency to relapse can be reduced by continued treatment with mood stabilisers $^{6}$ and psychoeducation. ${ }^{7}$ Nevertheless, naturalistic follow-up studies suggest that the progression of the diseases is not prevented in clinical practice with current available treatments. $^{1,2}$ Part of the explanation might be decreased adherence to mood stabilisers ${ }^{8}$ and delayed intervention with pharmacological and psychological treatment programmes. ${ }^{9,10} \mathrm{~A}$ number of randomised trials have investigated the effect of combined pharmacological and psychological interventions and various health-service interventions in bipolar disorder ${ }^{11,12}$ but none of the trials have specifically investigated the early stages of bipolar disorder. Observational studies suggest that early intervention may improve both course and outcome. Delay to first treatment is associated with more time depressed, greater severity of depression, more episodes, more days of ultradian cycling and fewer days euthymic. ${ }^{13}$ Patients with first-episode bipolar disorder present with better psychosocial function than patients with multiple episodes ${ }^{14}$ who rarely achieve functional recovery. ${ }^{15}$ The occurrence of multiple prior episodes seems to be a risk factor for non-response to a variety of pharmacological treatments. ${ }^{16}$ More specifically, response to lithium monotherapy has been

'See editorial, pp. 170-171, this issue. found to decrease with the occurrence of multiple prior episodes. ${ }^{17}$ Mood stabilisers prescribed for bipolar disorder may have neuroprotective abilities ${ }^{18,19}$ and patients may profit from psychoeducation before potential cognitive disturbances occur during the long-term course of illness. ${ }^{20}$ Further, an open-label pilot study showed that add-on psychological intervention compared with treatment as usual reduced depression, overall symptom severity and functional outcome in 40 patients following a first episode of psychotic mania. ${ }^{21}$

It has been claimed that specialised bipolar disorder units and programmes, such as the bipolar disorder programme at the Barcelona Hospital Clinic, are needed to improve outcome and advance research in bipolar disorder. ${ }^{22}$ The main advantage of specialised mood disorder clinics is that focused treatment programmes combining updated evidence-based pharmacological treatment with group psychological interventions such as group psychoeducation can be provided by a cross-disciplinary team of professionals that are specialised and scientifically up to date about bipolar disorder. In addition, the establishment of such clinics greatly increases the possibility of performing crossdisciplinary research in bipolar disorder. ${ }^{22}$ Nevertheless, it has never been investigated in a randomised trial whether treatment in such specialised bipolar disorder clinics improves the long-term outcome for people with bipolar disorder. The aim of the present randomised trial was to investigate whether treatment in a specialised mood disorder clinic, combining pharmacological treatment with group psychoeducation early in the course of illness among patients discharged from one of their first admissions to hospital for bipolar disorder, improves long-term 
outcome compared with standard psychiatric out-patient treatment. The trial design was pragmatic with very few exclusion criteria and investigated the effect among patients following admission to psychiatric hospital in The Capital Region of Denmark for the first, second or third time. This pragmatic design was chosen to obtain a high generalisability of results from the trial to clinical settings for treatment of patients early in the course of bipolar disorder. $^{23}$

\section{Method}

The trial protocol has been described in detail elsewhere. ${ }^{24} \mathrm{~A}$ summary of the design and method is presented here. The trial is registered at ClinicalTrials.gov: NCT00253071.

\section{Participants}

A total of 158 patients with a diagnosis of a single manic episode or bipolar disorder were included from seven (Psychiatric Centre Hvidovre, Psychiatric Centre Copenhagen, Rigshospitalet; Psychiatric Centre Amager, Psychiatric Centre Frederiksberg, Psychiatric Centre Copenhagen, Bispebjerg, Psychiatric Centre Gentofte, Psychiatric Centre Hillerød) out of the nine psychiatric wards in The Capital Region of Denmark during the period December 2005 to December 2009.

\section{Inclusion and exclusion criteria}

The inclusion criteria were: patients discharged from their first, second or third hospital admission from an in-patient psychiatric ward, with an ICD-10 diagnosis of single manic episode or bipolar disorder (ICD-10 code: DF $30.1-31.6)^{25}$ as the primary diagnosis. The vast majority had bipolar I disorder. Comorbidity with alcohol or substance misuse and other psychiatric disorders were allowed. The physicians at the psychiatric wards diagnosed the patients. Age was between 18 and 70 years of age. The patients were able and willing to give written and oral informed content. We excluded patients with moderate or severe dementia, with poor understanding of Danish, those under any kind of commitment (e.g. compulsory hospitalisation or treatment); or those without informed consent.

\section{Randomisation}

Patients were randomised 1:1 to the mood disorder clinic group or the standard care (control) group at the end of the index hospital admission while still in hospital. The Copenhagen trial unit conducted randomisation centrally according to a computergenerated allocation sequence to secure allocation concealment. Allocation was stratified for psychiatric centre and number of previous admissions to hospital, including the index hospital admission (one or two compared with three). The randomisation was carried out with a block size of 20 unknown to the investigators.

\section{Masking}

Masking of patients and the treating clinicians was not possible as patients were randomised to the mood disorder clinic $v$. standard out-patient treatment. The primary outcome was based on public register data using masking for the intervention. All other outcomes were assessed without masking to the intervention. Two researchers (L.V.K. and H.V.H.) carried out all statistical analyses before the primary outcome data were unmasked.

\section{Experimental intervention group}

Patients in the mood disorder clinic group were treated in a specialised out-patient clinic, The Copenhagen Affective Disorder Clinic, the Capital Region of Denmark at the Psychiatric Centre
Copenhagen, Copenhagen University Hospital, Rigshospitalet. The staff in the out-patient mood disorder clinic consist of full-time specialists in psychiatry with specific clinical experience and knowledge about the diagnosis and treatment of bipolar disorder as well as certified psychologists, nurses and a social worker with experience in bipolar disorder. The clinic offers combined intervention with evidence-based pharmacological treatment and group psychoeducation. Manuals for psychoeducation were developed, tested and revised in a pilot phase with inclusion of approximately 30 patients (the manuals, in Danish, are available from L.V.K. on request).

The final combined pharmacological and non-pharmacological intervention programme was as follows. The intervention programme lasted 2 years. According to the protocol, a medical doctor evaluated all patients in the clinic as early as possible following discharge from an in-patient admission and no later than 2 weeks after discharge as this is a vulnerable period. It is well known that the first weeks after discharge is a high-risk period for relapse and readmission to hospital, ${ }^{1}$ presumably because patients are not fully remitted at the time of discharge. Prior course of illness and effect of treatment was carefully recorded and diagnosis and treatment plans were re-evaluated and current pharmacological treatment adjusted in accordance with clinical status and with an approach very similar to the revised recommendations from the British Association for Psychopharmacology that were published in 2009. ${ }^{26}$ The focus was therefore on treatment with mood stabilisers, mainly lithium, valproate, lamotrigine and atypical antipsychotics. Often monotherapy was insufficient to obtain or maintain remission. When the diagnosis was mania, according to ICD-10 and evaluation with the Young Mania Rating Scale (YMRS), ${ }^{27}$ lithium or valproate was combined with an antipsychotic. When the diagnosis was depression, according to ICD-10 and evaluation with the 17-item Hamilton Rating Scale for Depression (HRSD-17), ${ }^{28}$ lithium or lamotrigine was combined with quetiapine. ${ }^{26}$ Antidepressants were only prescribed when remission could not be obtained in other ways, and in those cases treatment was mainly with selective serotonin reuptake inhibitors combined with one or two mood stabilisers. ${ }^{26}$

The physician at the mood disorder clinic followed the patients with regular appointments depending on their clinical status and needs. In addition, patients participated in three different sequential group sessions. The first group was a settling-in group for patients just discharged from hospital. Here the focus was on current clinical status and beliefs and experiences in relation to the recent admission. Patients stayed in this group until they were clinically stable and had at least partly remitted from depressive and manic symptoms (scores on HRSD-17 $<14$ and on YMRS <14), i.e. typically up to 6 months. When stable, the patients were transferred to the second and intermediary group, consisting of group psychoeducation. These group sessions lasted $1.5 \mathrm{~h}$ and were held every week for 12 consecutive weeks, followed by three additional booster sessions. In both groups, the focus was on knowledge and acceptance of having bipolar disorder, identifying depressive and manic symptoms from normal reactions, personal identity in relation to having bipolar disorder, risk situations, stress management, the need for sustained pharmacological maintenance treatment, adverse effects of treatment and identification of individual early warning signs of upcoming depressive and manic episodes. In addition, in some sessions cognitive-behavioural therapeutic approaches were included, focusing on cognitive distortions in identity and behaviour and to some extent on interindividual conflicts. Finally, the patients joined a 3-6 months discharge group that was a preparation for re-referral either to a general practitioner (GP), a private psychiatrist or to the community psychiatric centre, 
with the aim of identifying individual early warning signals and communicating these to the clinicians for a fast pharmacological re-evaluation or for an adequate behavioural response (for example to reduce social activities). Six to eight patients and two therapists (psychiatrist and psychologist or nurse) participated in each group.

\section{Control group}

The control group was offered standard care consisting of the standard out-patient mental health service routines in The Capital Region of Denmark, i.e. treatment with a GP, a private psychiatrist, at the local community mental health centre or a local psychiatrist associated with the discharging ward. Participation in the trial had no influence on the treatment offered to these patients. Psychopharmacological treatment in the control group, compared with treatment in the mood disorder clinic, was more likely to be based on the preferences of the individual physician than on national and international guidelines. Psychosocial treatment elements such as group psychoeducation or systematic individual psychoeducation was not offered, and contact with family was provided more infrequently and in a less intensive, non-systematic way compared with the mood disorder clinic.

\section{Main outcome measures}

The primary outcome measure was first readmission to the psychiatric ward after discharge from the index hospital admission. Data on readmission were obtained from the Danish Psychiatric Central Register that contains data on all in- and out-patient contacts to all psychiatric hospital-based services in Denmark. ${ }^{29}$ Since 1 January 1994, the ICD-10 has been used by the Register. ${ }^{25}$

The secondary and the tertiary outcomes were assessed using questionnaires that were mailed to all participants 1 and 2 years after discharge from the index hospital admission. The patients completed the Major Depression Inventory (MDI $)^{30}$ to identify relapse of a depressive episode. No questionnaire has been developed specifically for monitoring relapse of hypomanic/manic episodes and consequently the best option was to include the Mood Disorder Questionnaire (MDQ). ${ }^{31}$ According to standard definitions, a depressive episode was defined as a score of 21 or more on the MDI and a hypomanic/manic episode was defined as a score of 7 or more on the MDQ. In addition, patients were asked whether they used mood stabilisers (lithium or anticonvulsants), atypical antipsychotics and/or antidepressants.

The tertiary outcome was satisfaction with the intervention 1 and 2 years after discharge from the index hospital admission estimated by the Verona Service Satisfaction Scale adjusted for patients with affective disorder, the Verona Service Satisfaction Scale - Affective Disorder (VSSS-A). ${ }^{32}$ The VSSS-A includes, among others, 32 different items on satisfaction with care within seven different areas (overall satisfaction, professionals' skills and behaviour, information, access, efficacy, types of intervention, relatives' involvement). Patients rate each item on a five-point Likert scale (1, terrible; 2 , mostly dissatisfactory; 3 , mixed; 4, mostly satisfactory; 5 , excellent) making it possible to calculate a mean total score of satisfaction (between $0-5$ ).

For each variable, data on questionnaires were combined for the responses at 1 and 2 years into one combined measure.

\section{Costs}

Direct net costs were compared for treatment in a mood disorder clinic $v$. standard out-patient care. Only direct health-related costs resulting from psychiatric out-patient treatment and subsequent in-patient care were included. Other potential costs for local authorities, health services in general or indirect cost for society such as transferred income or decline in work capacity and productivity were not included.

Calculation of costs for treatment in the mood disorder clinic were based on the assumption that the case-load of patients per clinician was 20:1 in the clinic, which is the same as in a representative local community mental health centre in Copenhagen. ${ }^{30}$ The costs for patients treated in a representative local community mental health centre in Copenhagen was estimated from the model used in the Danish Health Technology Assessment report on preventive out-patient treatment in affective disorders (2006). ${ }^{33}$ In 2004 the treatment cost for one patient in a local mental health centre during 1 year was 3923 euros and with up-regulation to the index number price in 2012 adding $22.4 \%$, this corresponds to 4802 euros per year. Mean consultation fees per out-patient are standardised in Denmark to 63 euros for a private psychiatrist and 219 euros for a consultation for outpatient treatment at a local psychiatric hospital. ${ }^{31}$ The number of consultations for each patient with a private psychiatrist or the local psychiatric hospital was estimated to be a visit per month in a 2-year period.

Costs of in-patient care were based on the cumulated duration of all hospital admissions following randomisation. Danish Health Department figures for the cost of in-patient services in psychiatric hospitals is 439 euros per day, which is a conservative mean rate. $^{34}$

\section{Statistical analysis}

We planned a trial with 1 control patient per experimental patient, an accrual period of 4 years, and additional follow-up after the accrual interval of 2 years. We expected the median time to readmission in the standard care group to be $2-3$ years. If the true hazard ratio (HR) of patients treated in the mood disorder clinic relative to patients treated according to standard care is 0.60 , we needed to randomise 82 patients to each group to be able to reject the null hypothesis that the experimental and control survival curves are equal with a power of $80 \%$ and a type 1 error risk of 0.05 . The statistical analyses were conducted as intention-to-treat analyses. Regarding the primary outcome, time to the first readmission was estimated in a Kaplan-Meier plot, stopping at the date of death or end of study. The difference in cumulated prevention of readmission in the mood disorder clinical group and in the control group was tested in a log-rank test. Hazard ratios adjusted for age, gender, psychiatric centre and number of previous psychiatric admissions at baseline were calculated in Cox regression models. These covariates were chosen as they were included as stratification variables (psychiatric centre, number of previous psychiatric admissions) and/or based on previous evidence that they may affect the risk of readmission in bipolar disorder (age, gender, number of previous psychiatric admissions). ${ }^{1,35}$ Participants and non-participants were compared using register-based variables to evaluate whether participants in the trial were representative of patients with bipolar disorder discharged from their first, second or third hospital admission. We used SPSS 19.0 for Windows for the statistical analyses.

\section{Ethical considerations}

The trial was approved by the Danish Research Ethical Committee (KF 01 272130), covering all hospitals in the region, the Danish Data Protection Agency (CVR-nr. 11088037-29) and the Danish National Board of Health. There was written informed consent from all patients involved in the trial, including consent to participate in the trial and consent to publish, where appropriate. 
The participant's unique and personal identification number (CPR) was submitted to the Danish National Board of Health to link with data from the Danish Psychiatric Central Register ${ }^{36}$ and the Danish Medical Register on Vital Statistics. ${ }^{37}$

\section{Results}

According to the Danish Psychiatric Central Register, a total of 474 patients fulfilled the inclusion criteria as they had an ICD-10 diagnosis of a manic episode or bipolar disorder (F30-F31.9) at discharge from their first, second or third psychiatric hospital admission during the study period 1 December 2005 to 1 December 2009. Among these eligible patients, 158 patients were randomised, 72 patients to treatment in the mood disorder clinic v. 86 patients to standard out-patient treatment. The remaining 316 patients were either not assessed for participation in the trial $(80 \%)$ or excluded due to dementia, poor Danish, any kind of commitment (compulsory hospitalisation or treatment) or lack of informed consent. The 158 patients who participated in the trial did not differ from the 316 other potentially eligible patients regarding gender (female: $54.4 \%$ compared with $48.4 \% ; P=0.2$ ) but were younger (median 35.6 years (quartiles 27.7-47.1) compared with a median of 44.0 years (quartiles 33.0-57.0), $P<0.001)$.

The intervention in principle started at the date of discharge from the index hospital admission as patients before discharge were randomised to receive treatment in the mood disorder clinic $v$. standard treatment. Register-based data on readmission and death was $100 \%$ complete, i.e., available for all 158 included patients. No patient was lost to follow-up and no patient was excluded post-randomisation from the analyses.

The two intervention groups seemed reasonably well balanced regarding baseline variables (Table 1). Patients in the standard care group were treated at the local community mental health centre $(n=49,56.5 \%)$, by a private psychiatrist $(n=21,24.7 \%)$, by a local psychiatrist associated with the discharging ward $(n=33$, $15.3 \%)$ or by the GP $(n=3,3.5 \%)$. All patients were followed to the first event, a readmission to hospital, or to the date of death or emigration or to end of the study on 31 December 2011, whichever came first. One patient died and three patients emigrated during follow-up - all these patients were treated in the mood disorder clinic.

The follow-up period from the discharge date following randomisation was between 0 and 6 years with an average follow-up of 2.5 years (s.d.=1.7). In Fig. 1, it can be seen from the Kaplan-Meier curves that time to first psychiatric readmission was decreased for patients treated in the mood disorder clinic compared with patients who had standard treatment. Table 2 presents time to readmission for the two groups and a log-rank test confirmed that there was a statistically significant difference between the two groups $\left(\chi^{2}=4.49\right.$, d.f. $=1, P=0.034$; unadjusted $\mathrm{HR}=0.60,95 \%$ CI $0.37-0.97, P=0.034)$. When adjusted for the effect of age, gender, psychiatric centre and number of previous psychiatric admissions in a Cox regression model, the difference between the intervention groups remained statistically significant ( $\mathrm{HR}=0.60,95 \%$ CI $0.37-0.98, P=0.043)$.

A total of $26(36.1 \%)$ patients treated in the mood disorder clinic v. $47(54.7 \%)$ patients treated with standard care were readmitted (Table 2). The duration of first readmission following randomisation was shorter for those in the mood disorder clinic group compared with the standard care group, however, the difference was not statistically significant $(P=0.3$, mood disorder clinic $v$. standard care, median (quartiles): 12.0 (3.0-46.5) days $v$. 22.0 (4.8-54.8) days). Similarly, the mood disorder clinic group experienced a decreased number of total readmissions following randomisation, but the difference was not statistically significant $(P=0.11$; mood disorder clinic $v$. standard care, mean: 0.97 $($ s.d. = 1.74) v. $1.58($ s.d. $=2.57))$. The cumulated duration of all admissions following randomisation was significantly shorter for those in the mood disorder clinic group $(P=0.01$; mood disorder clinic $v$. standard care, median (quartiles): 33.0 (10.5-133.5) days v. 49.0 (21.0-127.5) days).

A total of $57(79.2 \%)$ of the 72 patients in the mood disorder clinic group and $46(53.5 \%)$ of the 86 patients in the standard care group completed and returned the mailed questionnaire 1 or 2 years after discharge from the index hospital admission $(P=0.001)$. Due to this difference in response rates, results regarding all the following analyses are reported unadjusted as well as adjusted for age, gender, psychiatric centre and number of previous psychiatric admissions (in multivariate analyses). According to the MDI, fewer patients relapsed into a depressive episode in the mood disorder clinic group $(n=25,35.1 \%)$ compared with the standard treatment group $(n=37,43.5 \%)$, however, the difference did not reach statistical significance (unadjusted $P=0.4$; adjusted $P=0.4$ ). Similarly, there was no statistically significant difference in relapse rates for a hypomanic or manic episode according to the MDQ (mood disorder clinic group $(n=45,62.9 \%)$, standard treatment group $(n=49$, $57.1 \%$ ), unadjusted $P=0.6$, adjusted $P=0.6$ ).

Satisfaction with treatment showed a statistically highly significant difference between patients in the mood disorder clinic

\begin{tabular}{|c|c|c|}
\hline & $\begin{array}{l}\text { Mood disorder clinic group } \\
\qquad(n=72)\end{array}$ & $\begin{array}{l}\text { Standard treatment group } \\
\qquad(n=86)\end{array}$ \\
\hline Female, $n(\%)$ & $44(61.1)$ & $42(48.8)$ \\
\hline Age at randomisation, years: median (quartiles) & $37.6(27.3-48.2)$ & $35.2(27.9-46.3)$ \\
\hline$\geqslant 11$ years of education, $n(\%)$ & $55(76.4)$ & $53(61.6)$ \\
\hline \multicolumn{3}{|l|}{ Employment, $n(\%)$} \\
\hline Employed & $50(69.4)$ & $43(50.0)$ \\
\hline Unemployed & $22(30.6)$ & $43(50.0)$ \\
\hline \multicolumn{3}{|c|}{ Patients with or without previous admission before index hospital admission, $n$ (\%) } \\
\hline Without & $41(56.9)$ & $40(46.5)$ \\
\hline With & $31(43.1)$ & $46(53.5)$ \\
\hline \multicolumn{3}{|l|}{ Centre, $n(\%)$} \\
\hline Hvidovre & 24 (33.3) & $30(34.9)$ \\
\hline Rigshospitalet & $25(34.7)$ & $25(29.1)$ \\
\hline Amager & 8 (11.1) & $11(12.8)$ \\
\hline Others & $15(20.8)$ & $20(23.3)$ \\
\hline
\end{tabular}




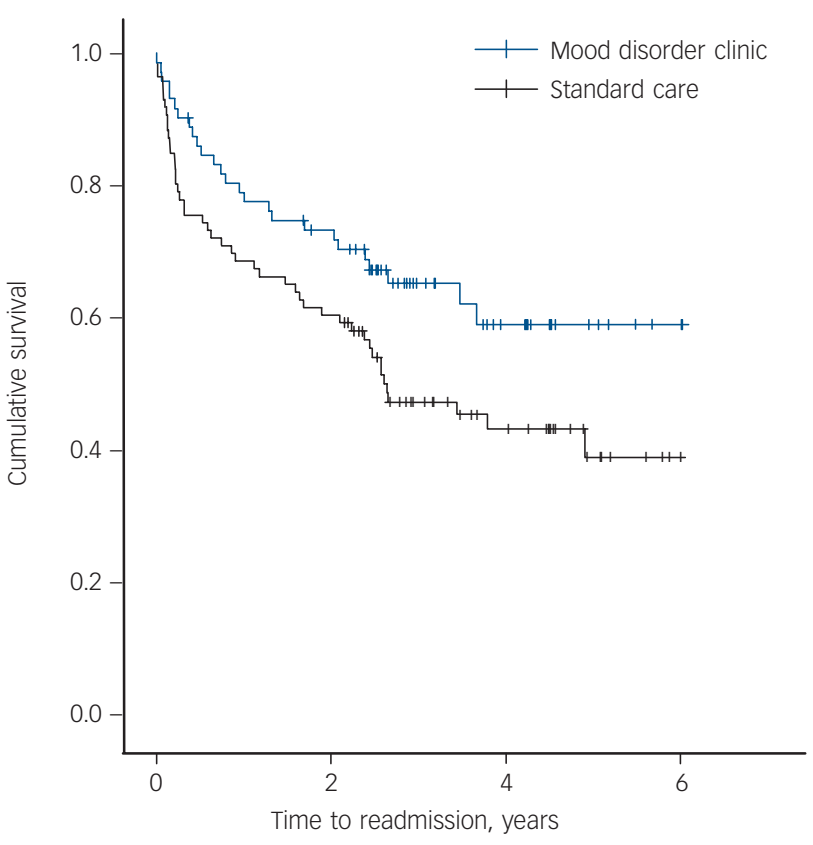

Fig. 1 Time to hospital readmission for patients treated in the mood disorder clinic $v$. standard out-patient care.

$v$. the standard care group (VSSS-A total score: $132.2($ s.d. $=16.9)$ v. 114.9 (s.d. $=31.6$ ), unadjusted $P=0.001$, adjusted $P=0.01$ ).

The mailed questionnaire included specific questions on consumed medication. The response rates regarding these specific questions were higher than the response rates regarding symptoms: $77.8 \%$ for antipsychotics, $80.4 \%$ for antidepressants and $92.4 \%$ for mood stabilisers (lithium or anticonvulsant). More patients in the mood disorder clinic $(n=42,59.0 \%)$ than in standard care $(n=28,32.4 \%)$ reported that they used a mood stabiliser (unadjusted $P=0.001$, adjusted $P=0.004$ ) or an antipsychotic $(n=43(59.5 \%)$ v. $n=30 \quad(34.9 \%)$, unadjusted $P=0.01$, adjusted $P=0.02)$ but there was no significant difference in use of antidepressants $(n=31(42.9 \%)$ v. $n=32(37.2 \%)$, unadjusted $P=0.5$, adjusted $P=0.8$ ).

Estimated direct costs are summarised in Table 3. The mean cost per patient treated in the mood disorder clinic was $9604 v$.
6604 euros per patient in standard out-patient care, a difference of 3000 euros. The additional costs for the mood disorder clinic were more than offset by reduced hospital admissions as the in-patient costs for patients in the mood disorder clinic group (14 487 euros) were only two-thirds of the amount for those in the standard care group (21511 euros). The costs of out-patient medication use represent only a small proportion of the overall costs. As can be seen from Table 3, the total direct net costs for treatment in the mood disorder clinic were 3194 euros less per patient than for standard care, corresponding to $11 \%$ of the costs for standard care.

Sensitivity analysis showed that the treatment offered in the mood disorder clinic could be up to $33 \%$ more expensive than standard out-patient treatment before it would become a more expensive option. Conversely, if all other parameters were equal, the reduction of in-patient days used by patients treated in the mood disorder clinic had to be less than 9 days or a reduction of $18 \%$ before treatment in the mood disorder clinic was more expensive than standard out-patient care.

\section{Discussion}

We randomised patients, who were in the early course of severe bipolar disorder, to either out-patient treatment in a mood disorder clinic or standard care (i.e. treatment by a GP, a private psychiatrist or at the local community mental health centre), as they were discharged from their first, second or third psychiatric hospital admission with a diagnosis of mania/bipolar disorder. The main outcome was readmission to a psychiatric hospital and the follow-up period from the discharge date following randomisation was between 0 and 6 years with an average follow-up of 2.5 years (s.d.=1.7). We found that out-patient treatment in the mood disorder clinic resulted in a $40 \%$ reduction in risk of readmission to hospital, which represents a substantial decrease. Similarly, the duration of first readmission (median (quartiles): 12.0 (3.0-46.5) days v. $22.0(4.8-54.8)$ days) and the cumulated duration of all readmissions (median (quartiles): 33.0 (10.5-133.5) days v. 49.0 (21.0-127.5) days) following randomisation were shorter for patients in the mood disorder clinic group compared with the standard care group (although the difference was not statistically significant for the former comparison). Patients in the mood disorder clinic group more often used a mood stabiliser or an antipsychotic, and satisfaction

Table 2 Comparison of time to readmission for patients treated in the mood disorder clinic $v$. standard care

\begin{tabular}{|c|c|c|c|c|c|c|}
\hline \multirow[b]{2}{*}{ Treatment } & \multirow[b]{2}{*}{$n$} & \multirow{2}{*}{$\begin{array}{l}\text { Events (readmissions) } \\
n(\%)\end{array}$} & \multirow{2}{*}{$\begin{array}{l}\text { Events censored because of } \\
\text { death or end of trial, } n(\%)\end{array}$} & \multirow{2}{*}{$\begin{array}{l}\text { Years of post-randomisation } \\
\text { survival time, mean }(95 \% \mathrm{Cl})\end{array}$} & \multicolumn{2}{|c|}{ Log rank test } \\
\hline & & & & & $\chi^{2}$ (d.f.) & $P$ \\
\hline Mood disorder clinic group & 72 & $26(36.1)$ & $46(63.9)$ & $4.1(3.5-4.7)$ & 4.49 (1) & 0.034 \\
\hline Standard care group & 86 & $47(54.7)$ & 39 (45.3) & $3.2(2.7-3.8)$ & & \\
\hline
\end{tabular}

\begin{tabular}{|lccc|}
\hline Table 3 Two-year treatment mean costs per patient in the mood disorder clinic v. standard care (euros) \\
\hline Mood disorder clinic & $\begin{array}{c}\text { Mood disorder } \\
\text { clinic }\end{array}$ & $\begin{array}{c}\text { Standard } \\
\text { care }\end{array}$ & $\begin{array}{c}\text { Cost difference between mood } \\
\text { disorder clinic and standard care }\end{array}$ \\
\hline Mental health centre & 9604 & - & -5426 \\
\hline Private psychiatrist & - & 5426 & -374 \\
\hline Out-patient treatment at the local psychiatric hospital & - & 874 & -804 \\
\hline Medicine & 1862 & 1032 & -730 \\
\hline In-patient cost (total follow-up period) & 14487 & 21511 & -3194 \\
\hline Net cost & 25953 & 29147 & -3024 \\
\hline
\end{tabular}


with treatment was more prevalent than among patients who received standard care. Data on secondary outcomes, depressive and manic episodes were flawed by the low response rates to the mailed questionnaires.

It should be noted that patients included in the trial had the most severe bipolar disorder that had led to psychiatric hospital admissions. Consequently, as patients were recruited following their first admission, the median age of 35.6 years at inclusion in the study was rather high, although with a substantial variation; $25 \%$ were below 28 years and $25 \%$ above 47 years of age at inclusion. This relatively high age of the sample is also found in two other studies recruiting patients following a first admission (mean age 31.4 years $($ s.d. $=12.9)$ and 38.4 years $($ s.d. $=12.6$ ) respectively). ${ }^{38,39}$ There may be three major reasons why such samples have a higher mean age. Patients with onset of a severe or more abrupt first manic episode resulting in admission to hospital may have a higher age at onset than patients with milder first episodes. There may be a delay in the diagnosis of bipolar disorder, as early episodes may be mistaken for transient psychosis, reaction to stress/adjustment disorder, or alcohol or other substance misuse. ${ }^{40}$ Moreover, patients with bipolar disorder who present with a depressive episode or recurrent depressive episodes as their first episodes represent as much as half of all patients being admitted to hospital. ${ }^{39-41} \mathrm{~A}$ prior study from Denmark showed that there was 5.0 years on average between onset of a first episode and first hospital admission with a diagnosis of bipolar disorder and 3.6 years between first pharmacological treatment and first hospital admission for bipolar disorder. ${ }^{42}$ Notably, when the first episode is a depressive episode, time to diagnosis of bipolar disorder will be delayed in prospective studies such as the present study, as patients initially will be classified as having a unipolar disorder. Thus, a proportion of the patients in our sample received treatment for depressive episodes or even milder-to-moderate manic episodes for a period before admission to hospital. In summary, despite the relatively old age of our patients, we believe that the study included patients who were early in the course of the more severe type of bipolar disorder.

Time to (re)admission to hospital as an outcome has been criticised as reductionistic. However, it benefits from being consistently recorded and may have high face validity as admission to hospital reflects serious relapse of the illness. ${ }^{43}$ There was no formal difference in the circumstances relating to decisions about readmitting patients, or practical ways of doing so (i.e. the procedures for hospitalisation), between the mood disorder clinic and the psychiatric centres offering standard care. The difference in readmission rates between the two interventions were not explained by an increased threshold for admission by the physicians in the mood disorder clinic compared with those in standard care, as the difference in readmission rates continued to increase following the end of the 2-year treatment period in the clinic, at which time patients in the mood disorder clinic were referred to treatment in standard care settings (see Fig.1).

\section{Limitations}

It was not possible to mask patients or treating clinicians to the study groups because of the nature of the interventions used. Patients in the experimental group received a well-defined intervention programme combining evidence-based pharmacological treatment and manualised group psychoeducation. It is not possible to distinguish between the effects of these pharmacological and psychological treatments in the trial. Patients in the mood disorder clinic reported using more mood stabilisers and atypical antipsychotics than patients in standard care but there was no difference in the use of antidepressants; in the mood disorder clinic group, medication use was in accordance with the recommendations from the British Association for Psychopharmacology. ${ }^{26}$ Thus, patients in the mood disorder clinic adhered more to medication, in ways recommended in current guidelines.

On the other hand, it is likely that the patients in the control group received very different interventions and that these interventions varied from being broad, competent and prolonged, to being shorter and sporadic. Patients were mainly treated in community psychiatric centres $(56.5 \%)$ and to a lesser extent by private specialists in psychiatry $(24.7 \%)$ or a local psychiatrist associated with the discharging ward (15.3\%). Data on the number or frequency of out-patient visits were not available for the two intervention groups.

The trial did not include a rigorous assessment during the follow-up period of the secondary outcome measures, i.e. relapse of affective episodes in a face-to-face research-based interview, but only suboptimal assessment based on patients' self-reported responses to the MDI and MDQ. Furthermore, the response rate to the MDI and MDQ was relatively low (65.2\%) increasing the possibility of selection bias related to the answers between the intervention groups.

The economic analysis is subject to some uncertainty as it was not possible to detect all individual costs for all services but only the mean costs per patients treated in the mood disorder clinic or in standard treatment. Indirect costs for patients and their families, health resource utilisation in general, or indirect cost for society such as unemployment or absenteeism from work were not included in the analyses. Nevertheless, it is more likely that these costs were lower for patients treated in the mood disorder clinic compared with those treated in standard care. A study from the UK has shown that indirect costs account for approximately $86 \%$ of the total costs for bipolar disorder ${ }^{44}$ and if treatment in the mood disorder clinic had just a small positive effect in increasing work capacity and productivity, it would have a great impact on the total costs for society.

\section{Generalisability}

Pragmatic trials, such as the present trial, are designed to measure effectiveness; that is, whether an intervention works when used in usual conditions of care. To ensure applicability in a wide range of usual care settings, pragmatic trials should include all kinds of participants to whom potentially the intervention may be offered in the real world, if its effectiveness is established. The trial included a moderate number of patients with bipolar disorder with all kinds of symptoms and comorbidities and there were very few exclusion criteria.

\section{Perspective}

It has not previously been investigated whether treatment in specialised mood disorder clinics decreases the risk of admissions to hospital for patients with bipolar disorder. However, a few studies have investigated the effect of more specific group-based interventions offered in bipolar disorder units such as psychoeducation or psychosocial intervention in relation to hospital readmissions as an outcome. Colom et al showed that treatment with 21 sessions of group psychoeducation compared with non-specific group meetings did not reduce the number of patients who required admissions but there was a significant reduction in the number of hospital admissions per patient during the 2-year follow-up. ${ }^{7,12}$ Their trial showed that group psychoeducation $v$. unstructured group support saved health resources, particularly those related to admissions to hospital. ${ }^{45}$ 
In-patient care accounted for $40 \%$ of the estimated total cost in the control group but only about $15 \%$ in the psychoeducation group. In the present trial, in-patient care accounted for $75.3 \%$ of the estimated total cost in the standard care group $v .55 .8 \%$ in the mood disorder clinic group (based on data from Table 3). Thus, costs related to in-patient care were relatively higher in our trial, reflecting the high readmission rates as patients were unstable because they had just been discharged from hospital when included in our trial, whereas they were in a remitted stable period when included in the Barcelona trial. ${ }^{45}$

We investigated the effect of a comprehensive programme in a mood disorder clinic combining a specific focus on evidencebased pharmacological treatment with group psychoeducation once a week for 12 weeks, followed by three additional booster sessions. The focus on evidence-based treatment is reflected in the more frequent use of mood stabilisers and antipsychotics among patients treated in the clinic. The effect of the 2-year programme offered in the mood disorder clinic seems to extend to the period after discharge from the clinic as illustrated by Fig. 1, as the difference in the rate of readmission to hospital continued to increase after the 2-year treatment period in the clinic, and as also illustrated by the decreased cumulative duration of all admissions for patients treated in the clinic. This sustained effect of the 2-year treatment intervention in the mood disorder clinic is in accordance with the long-lasting prophylactic effects found in the 5-year follow-up of group psychoeducation $v$. nonstructured group in the Barcelona Hospital Clinic, ${ }^{46}$ although the designs of the two studies are very different.

Figure 1 further illustrates that the rate of readmission is especially high in the weeks and months following discharge and furthermore that the two curves separate soon after randomisation; emphasising the crucial need for contact with the out-patient facility within 2 weeks of discharge.

The findings of the present randomised trial add to the findings from observational studies that it is possible with early and sustained pharmacological and psychological treatment, like that offered in a mood disorder clinic, to improve the long-term course of illness in bipolar disorder. ${ }^{13,16,17}$ These findings suggest that more focus should be put on early out-patient intervention among patients with severe mania/bipolar disorder. The staff resources and patient volume of a specialised mood disorder clinic such as the Copenhagen Affective Disorder Clinic or the Barcelona Hospital Clinic secure and facilitate experiences with up-to-date and evidence-based treatments.

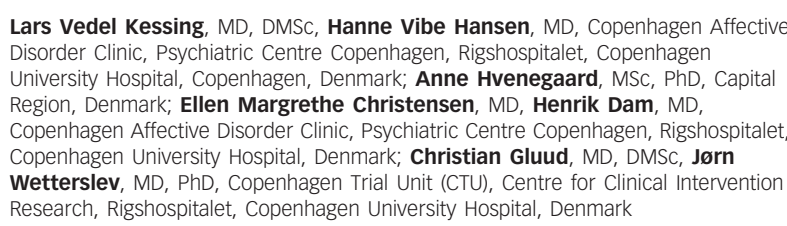

Correspondence: Lars Vedel Kessing, Psychiatric Centre Copenhagen, Rigshospitalet, Copenhagen University Hospital, Blegdamsvej 9, DK 2100 copenhagen Ø, Denmark. Email: lars.vedel.kessing@regionh.dk

First received 23 Apr 2012, final revision 16 Aug 2012, accepted 28 Nov 2012

\section{Funding}

The study is supported by the Lundbeck Foundation and by the Research Foundation of the Hovedstadens Sygehusfællesskab (The Capital Hospital Corporation).

\section{Acknowledgements}

Members of the EIA Trial Group also included: Klaus Damgård Jakobsen, Ejnar Bundgaard Larsen, Psychiatric Centre Hvidovre; Martin Balslev Jørgensen, Maj Vinberg, Rikke Engel,
Psychiatric Centre Copenhagen, Rigshospitalet; Flemming Mørkeberg Nilsson, Nana Hengstenberg, Psychiatric Centre Amager; Birgitte Bjerg Bendsen, Psychiatric Centre Frederiksberg; Hans Mørch Jensen, Psychiatric Centre Copenhagen, Bispebjerg; Rie Lambæk Mikkelsen, Psychiatric Centre Gentofte; Birgit Straasø, Jens Abraham, Psychiatric Centre Hillerød.

\section{References}

1 Kessing LV, Hansen MG, Andersen PK. Course of illness in depressive and bipolar disorders. Naturalistic study, 1994-1999. Br J Psychiatry 2004; 185 372-7.

2 Kessing LV, Hansen MG, Andersen PK, Angst J. The predictive effect of episodes on the risk of recurrence in depressive and bipolar disorders a life-long perspective. Acta Psychiatr Scand 2004; 109: 339-44.

3 Tohen M, Hennen J, Zarate Jr CM, Baldessarini RJ, Strakowski SM, Stoll AL, et al. Two-year syndromal and functional recovery in 219 cases of firstepisode major affective disorder with psychotic features. Am J Psychiatry 2000; 157: 220-8.

4 Torres IJ, Boudreau VG, Yatham LN. Neuropsychological functioning in euthymic bipolar disorder: a meta-analysis. Acta Psychiatr Scand Suppl 2007; 434: $17-26$.

5 Kessing LV, Nilsson FM. Increased risk of developing dementia in patients with major affective disorders compared to patients with other medical illnesses. J Affect Disord 2003; 73: 261-9.

6 Geddes JR, Goodwin GM, Rendell J, Azorin JM, Cipriani A, Ostacher MJ, et al. Lithium plus valproate combination therapy versus monotherapy for relapse prevention in bipolar I disorder (BALANCE): a randomised open-label trial. Lancet 2010; 375: 385-95.

7 Colom F, Vieta E, Martinez-Aran A, Reinares M, Goikolea JM, Benabarre A, et al. A randomized trial on the efficacy of group psychoeducation in the prophylaxis of recurrences in bipolar patients whose disease is in remission. Arch Gen Psychiatry 2003; 60: 402-7.

8 Kessing LV, Sondergard L, Kvist K, Andersen PK. Adherence to lithium in naturalistic settings: results from a nationwide pharmacoepidemiological study. Bipolar Disord 2007; 9: 730-6.

9 Macneil CA, Hallam K, Conus P, Henry L, Kader L, Berk M. Are we missing opportunities for early intervention in bipolar disorder? Expert Rev Neurother 2012; 12: 5-7.

10 Macneil CA, Hasty MK, Berk M, Henry L, Evans M, Redlich C, et al. Psychological needs of adolescents in the early phase of bipolar disorder: implications for early intervention. Early Interv Psychiatry 2011; 5: 100-7.

11 Nierenberg AA, Ostacher MJ, Borrelli DJ, Iosifescu DV, Perlis RH, Desrosiers A, et al. The integration of measurement and management for the treatment of bipolar disorder: a STEP-BD model of collaborative care in psychiatry. J Clin Psychiatry 2006; 67 (suppl 11): 3-7.

12 Colom F, Berk L. Psychoeducation as a core element of psychological approaches for bipolar disorder. In Bipolar Disorder: Clinical and Neurobiological Foundations (eds LN Yatham, M Maj): 412-21. John Wiley \& Sons, 2010

13 Post RM, Leverich GS, Kupka RW, Keck Jr PE, McElroy SL, Altshuler LL, et al. Early-onset bipolar disorder and treatment delay are risk factors for poor outcome in adulthood. J Clin Psychiatry 2010; 71: 864-72.

14 Rosa AR, Gonzalez-Ortega I, Gonzalez-Pinto A, Echeburúa E, Comes M, Martínez-Àran A, et al. One-year psychosocial functioning in patients in the early vs. late stage of bipolar disorder. Acta Psychiatr Scand 2012; 125: 335-41.

15 Rosa AR, Reinares M, Amann B, Popovic D, Franco C, Comes M, et al. Six-month functional outcome of a bipolar disorder cohort in the context of a specialized-care program. Bipolar Disord 2011; 13: 679-86.

16 Post RM, Fleming J, Kapczinski F. Neurobiological correlates of illness progression in the recurrent affective disorders. J Psychiatr Res 2012; 46: 561-73.

17 Kessing LV, Hellmund G, Andersen PK. Predictors of excellent response to lithium: results from a nationwide register-based study. Int Clin Psychopharmacol 2011; 26: 323-8.

18 Manji HK, Moore GJ, Chen G. Clinical and preclinical evidence for the neurotrophic effects of mood stabilizers: implications for the pathophysiology and treatment of manic-depressive illness. Biol Psychiatry 2000; 48: 740-54.

19 Fountoulakis KN, Vieta E, Bouras C, Notaridis G, Giannakopoulos P, Kaprinis $\mathrm{G}$, et al. A systematic review of existing data on long-term lithium therapy: neuroprotective or neurotoxic? Int J Neuropsychopharmacol 2008; 11: $269-87$.

20 Berk M, Hallam K, Lucas N, Hasty M, McNeil CA, Conus $\mathrm{P}$, et al. Early intervention in bipolar disorders: opportunities and pitfalls. Med J Aust 2007; 187: S11-4 
21 Macneil CA, Hasty M, Cotton S, Berk M, Hallam K, Kader L, et al. Can a targeted psychological intervention be effective for young people following a first manic episode? Results from an 18-month pilot study. Early Interv Psychiatry 2012; 6: 380-8.

22 Vieta E. Bipolar units and programmes: are they really needed? World Psychiatry 2011; 10: 152

23 Zwarenstein M, Treweek S, Gagnier JJ, Altman DG, Tunis S, Haynes B, et al. Improving the reporting of pragmatic trials: an extension of the CONSORT statement. BMJ 2008; 337: a2390.

24 Kessing LV, Hansen HV, Christensen EM, Dam H, Gluud C, Wetterslev J. The effects of centralised and specialised combined pharmacological an psychological intervention compared with decentralised and non-specialised treatment in the early course of severe unipolar and bipolar affective disorders-design of two randomised clinical trials. Trials 2011; 12: 32.

25 World Health Organization. Klassifikation af sygdomme. Systematisk Del [International Statistical Classification of Diseases and Health Related Problems (10th rev, Danish edn)]. Munksgaard, 1993.

26 Goodwin GM. Evidence-based guidelines for treating bipolar disorder: revised second edition - recommendations from the British Association for Psychopharmacology. J Psychopharmacol 2009; 23: 346-88.

27 Young RC, Biggs JT, Ziegler VE, Meyer DA. A rating scale for mania: reliability, validity and sensitivity. Br J Psychiatry 1978; 133: 429-35.

28 Hamilton M. Development of a rating scale for primary depressive illness. Br J Clin Psychol 1967; 6: 278-96.

29 Munk-Jorgensen P, Mortensen PB. The Danish Psychiatric Central Register. Dan Med Bull 1997; 44: 82-4.

30 Olsen LR, Jensen DV, Noerholm V, Martiny K, Bech P. The internal and external validity of the Major Depression Inventory in measuring severity of depressive states. Psychol Med 2003; 33: 351-6.

31 Hirschfeld RM, Williams JB, Spitzer RL, Calabrese JR, Flynn L, Keck Jr PE, et al. Development and validation of a screening instrument for bipolar spectrum disorder: the Mood Disorder Questionnaire. Am J Psychiatry 2000; 157 1873-5.

32 Kessing LV, Hansen HV, Ruggeri M, Bech P. Satisfaction with treatment among patients with depressive and bipolar disorders. Soc Psychiatry Psychiatr Epidemiol 2006; 41: 148-55.

33 Kessing LV, Hansen HV, Hougaard E, Hvenegaard A, Albæk J. Preventive Outpatient Treatment in Affective Disorders. Results from a Health Tecnology
Assessment (HTA). National Board of Health, Danish Centre for Evaluation and Health Tecnology Assessment, 2006.

34 Danish Ministry of Health. Rate Guidance 2012. Danish Ministry of Health, 2012 (http://www.sum.dk).

35 Kessing LV. Recurrence in affective disorder. II. Effect of age and gender. Br J Psychiatry 1998; 172: 29-34.

36 Munk-Jorgensen P, Mortensen PB. The Danish Psychiatric Central Register Dan Med Bull 1997; 44: 82-4.

37 Danish National Board of Health (Sundhedsstyrelsen). Cause of Death in Denmark. Sundhedsstyrelsen, 1992

38 Khalsa HM, Salvatore P, Hennen J, Baethge C, Tohen M, Baldessarini RJ. Suicidal events and accidents in 216 first-episode bipolar I disorder patients: predictive factors. J Affect Disord 2008; 106: 179-84.

39 Perugi G, Micheli C, Akiskal HS, Madaro D, Socci C, Quilici C, et al. Polarity of the first episode, clinical characteristics, and course of manic depressive illness: a systematic retrospective investigation of 320 bipolar I patients. Compr Psychiatry 2000; 41: 13-8.

40 Kessing LV. Diagnostic stability in bipolar disorder in clinical practise as according to ICD-10. J Affect Disord 2005; 85: 293-9.

41 Goodwin FK, Jamison KR. Manic Depressive IIIness. Bipolar Disorder and Recurrent Depression (2nd edn). Oxford University Press, 2007.

42 Kessing LV. Validity of diagnoses and other register data in patients with affective disorder. Eur Psychiatry 1998; 13: 392-8.

43 Burns T. End of the road for treatment-as-usual studies? Br J Psychiatry 2009; 195: 5-6

44 Gupta RD, Guest JF. Annual cost of bipolar disorder to UK society. Br J Psychiatry 2002; 180: 227-33.

45 Scott J, Colom F, Popova E, Benabarre A, Cruz N, Valenti M, et al. Long-term mental health resource utilization and cost of care following group psychoeducation or unstructured group support for bipolar disorders: a cost-benefit analysis. J Clin Psychiatry 2009; 70: 378-86.

46 Colom F, Vieta E, Sánchez-Moreno J, Palomino-Otiniano R, Reinares $\mathrm{M}_{\text {, }}$ Goikolea JM, et al. Group psychoeducation for stabilised bipolar disorders: 5-year outcome of a randomised clinical trial. Br J Psychiatry 2009; 194: $260-5$. 